07,01

\title{
Зависимость от давления коэффициентов упругости алюминий-магниевого сплава AMg6 и нанокомпозитного сплава $n-\mathrm{Mg} 6 / \mathrm{C}_{60}$
}

\author{
(C) В.М. Прохоров ${ }^{1}$, Е.Л. Громницкая ${ }^{2}$ \\ ${ }^{1}$ Технологический институт сверхтвердых и новых углеродных материалов, \\ Москва, Троицк, Россия \\ ${ }^{2}$ Институт фризики высоких давлений РАН, \\ Москва, Троицк, Россия \\ E-mail: pvm@tisnum.ru
}

(Поступила в Редакцию 31 октября 2017 г.)

\begin{abstract}
Приведены результаты измерений ультразвуковым методом зависимости коэффициентов упругости второго порядка поликристаллического алюминий-магниевого сплава $\mathrm{AMg} 6$ и приготовленного на его основе нанокомпозита $n$ - $\mathrm{AMg} 6 / \mathrm{C}_{60}$ от гидростатического давления до $1.6 \mathrm{GPa}$. Ультразвуковые измерения были проведены на пьезометрической установке высокого давления типа поршень-цилиндр. Установленные в работе значения производных по давлению коэффициентов упругости второго порядка сравнивались с рассчитанными по значениям коэффициентов упругости третьего порядка, которые были ранее определены методом Терстона-Браггера. С привлечением литературных данных установлены зависимости производных по давлению коэффициентов упругости второго порядка сплава AMg6 от содержания $\mathrm{Mg}$ и наноструктурирования.
\end{abstract}

Работа выполнена с использованием оборудования ЦКП „Исследования наноструктурных, углеродных и сверхтвердых материалов“ (ЦКП ФГБНУ ТИСНУМ), поддержанного Минобрнауки России в рамках выполнения соглашения № 14.593.21.0007 от 28.08.2017, ID RFMEFI59317X0007.

DOI: $10.21883 /$ FTT.2018.04.45690.300

\section{1. Введение}

Линейные упругие характеристики, определяемые коэффициентами упругости второго порядка $C_{i j k l}$ (КУВП), и нелинейные упругие характеристики, определяемые коэффициентами упругости третьего порядка $C_{i j k q r}$ (КУТП) и производными по давлению КУВП - это важные физические параметры материалов. КУВП служат параметрами линейной зависимости деформации от напряжения в твердом теле, описываемой законом Гука. КУТП являются коэффициентами разложения упругой энергии при деформации в третьей степени и отражают отклонения от линейной зависимости закона Гука. КУТП количественно описывают ангармонические свойства кристаллической решетки: тепловое расширение, взаимодействие фононов, высокочастотное поглощение ультразвука. КУТП также используются для анализа взаимодействия акустических волн конечной амплитуды в твердых телах и как диагностический параметр несовершенства (дефектности) структуры твердых тел [1,2]. Производная по давлению объемного модуля упругости - важный параметр уравнения состояния твердого тела. Производные по давлению КУВП могут быть выражены через КУТП и определены непосредственно и с хорошей точностью, путем измерения зависимости КУВП от давления.

Широко распространенный литой поликристаллический сплав алюминия $\mathrm{AMg} 6$ принадлежит к си- стеме $\mathrm{Al}-\mathrm{Mg}-\mathrm{Mn}$ (включает: до 93.68\% алюминия, $5.8-Z 6.8 \%$ магния, 0.5-0.8\% марганца и прочие примеси) и относится к наиболее прочным сплавам системы алюминий-магний $[3,4]$. Он характеризуется высокой технологической пластичностью, а также относительно высокими пределом прочности $\left(\sigma_{b}=110-310 \mathrm{MPa}\right)$ и текучести $\left(\sigma_{0,2}=40-160 \mathrm{MPa}\right)$, по сравнению с другими алюминиевыми сплавами в отожженном состоянии. Его высокие характеристики пластичности, как при комнатной, так и при повышенных температурах, высокая коррозионная стойкость в различных средах, в том числе и в морской воде, объясняют широкое применение этого сплава в различных областях промышленности. Сплавы этого семейства могут упрочняться только холодной пластической деформацией.

\section{2. Постановка задачи}

Механические и нелинейные упругие характеристики сплава AMg6 исследованы в ряде недавних работ [5-9], в которых также изучалось влияние наноструктурирования сплава на эти характеристики. Было установлено, что значения КУТП в сплаве AMg6 и его нанокомпозите $n$-AMg6/C 60 , отличаются в разы или даже в десятки раз. Так, например, величина коэффициента $C_{111}$ в сплаве $\mathrm{AMg} 6$ примерно в полтора раза больше величины этого коэффициента в образцах нанокомпозитного сплава $n$-AMg6/C 60 . Это указывает на то, что КУТП более 
Таблица 1. Усредненные значения КУТП сплава AMg6 и нанокомпозита $n$-AMg6/C 60 (в GPa)

\begin{tabular}{c|c|c|c|c|c|c}
\hline Alloys & $C_{111}( \pm 240)$ & $C_{112}( \pm 120)$ & $C_{123}( \pm 70)$ & $v_{1}=C_{123}( \pm 70)$ & $v_{2}=C_{144}( \pm 20)$ & $v_{3}=C_{456}( \pm 10)$ \\
\hline AMg6 & -3420 & -1310 & -860 & -860 & -230 & -150 \\
$n$-AMg6/C60 & -2770 & -980 & -680 & -680 & -150 & -150
\end{tabular}

чувствительны к химическому составу, структуре, стехиометрии материала, чем КУВП, а наноструктурирование материала приводит к значительным изменениям как пределов прочности и текучести, так и нелинейных упругих характеристик - КУТП и нелинейного акустического параметра [8-9].

Изотропное твердое тело характеризуется двумя независимыми КУВП $-C_{11}$ и $C_{44}$ (или модулями Ламе $\lambda=C_{11}-2 C_{44}$ и $\left.\mu=C_{44}\right)$ и тремя КУТП $-C_{111}, C_{112}$ и $C_{123}$ (или модулями третьего порядка $v_{1}, v_{2}, v_{3}$ ) [10]. Остальные КУВП и КУТП изотропного твердого тела выражаются через эти независимые коэффициенты упругости.

Экспериментально КУВП определяются, в большинстве случаев, путем измерений скоростей продольных и сдвиговых объемных акустических волн (ОАВ), а КУТП по измерениям зависимости скоростей ОАВ от гидростатического сжатия (давления) и/или одноосного сжатия. В экспериментах по гидростатическому сжатию непосредственно определяются производные по давлению КУВП: $\partial C_{11} / \partial P$ и $\partial C_{44} / \partial P$. Для вычисления 3-х независимых КУТП измерения в гидростатических условиях необходимо дополнить измерениями в условиях одноосного сжатия. С другой стороны, для определения трех независимых КУТП $C_{111}, C_{112}, C_{123}$ в изотропном твердом теле достаточно проведение трех независимых измерений зависимости скоростей ОАВ от величины одноосного сжатия $\mathbf{P}$ при следующем взаимном расположении единичных векторов М (вектор направления одноосного сжатия), $\mathbf{N}$ (вектор направления распространения ОАВ) и $\mathbf{U}$ (вектор поляризации ОАВ):

$$
\mathbf{N}\|\mathbf{U} \perp \mathbf{M}, \quad \mathbf{N} \perp \mathbf{U}\| \mathbf{M}, \quad \mathbf{N} \perp \mathbf{U} \perp \mathbf{M} .
$$

Направление одноосного сжатия при всех измерениях должно быть перпендикулярно направлению распространения упругой волны [11]. Для повышения точности измерений часто проводятся измерения для более чем трех комбинаций направлений единичных векторов $\mathbf{M}, \mathbf{N}, \mathbf{U}$.

В [9] для определения трех независимых КУТП методом Терстона-Браггера [10], в образцах исследуемых материалов были измерены зависимости относительного изменения скорости $(\Delta V / V)$ продольных и сдвиговых $\mathrm{OAB}$ от величины одноосного сжатия $\mathbf{P}$ (до 3 и $6 \mathrm{MPa}$, соответственно) при различных направлениях единичных векторов N, U и М. Результаты этих измерений воспроизведены в табл. 1 .

Для верификации установленных в [9] значений для КУТП сплавов $\mathrm{AMg} 6$ и $n-\mathrm{AMg} 6 / \mathrm{C}_{60}$ мы, в данной работе, провели измерения зависимости скоростей продольной и сдвиговой ОАВ от гидростатического давления, что дало возможность напрямую определить производные по давлению КУВП и сравнить их значения с рассчитанными по значениям КУТП, представленными в табл. 1.

\section{3. Производные по давлению КУВП}

Для расчетов производных по давлению КУВП использовались соотношения, полученные в [12], в виде

$$
\begin{aligned}
& -3 B \partial C_{11} / \partial P=C_{11}+C_{111}+2 C_{112} ; \\
& -3 B \partial C_{12} / \partial P=C_{12}+C_{123}+2 C_{112} ; \\
& -3 B \partial C_{44} / \partial P=C_{44}+C_{144}+2 C_{166} ; \\
\partial B / \partial P=( & \left.\partial C_{11} / \partial P-4 / 3 \partial C_{44} / \partial P\right) \\
= & -\left(C_{11}+2 C_{12}+C_{111}+6 C_{112}+2 C_{123}\right) / 9 B,
\end{aligned}
$$

где $B=\left(C_{11}+2 C_{12}\right) / 3-$ объемный модуль, $C_{166}=\left(C_{111}-C_{112}\right) / 4, C_{144}=\left(C_{112}-C_{123}\right) / 2$.

Также, производные по давлению КУВП можно выразить через модули второго и третьего порядков, согласHO $[10]$

$$
\begin{aligned}
-B \partial B / \partial P & =\left(v_{1}+2 v_{2}+8 / 9 v_{3}\right), \\
-B \partial C_{44} / \partial P & =\left(v_{2}+4 / 3 v_{3}+\lambda+\mu\right) .
\end{aligned}
$$

Результаты расчетов производных по давлению КУВП сплавов $\mathrm{AMg6}$ и $n-\mathrm{AMg6} / \mathrm{C}_{60}$ по формулам (1), на основе данных табл. 1, представлены в табл. 2.

Из табл. 2 видно, что наноструктурирование приводит к снижению рассчитанных из КУТП значений производных по давлению. Это обусловлено снижением значений КУТП в этих материалах. (Как отмечалось в $[8,9]$, и, как следует из анализа литературных данных, значения КУВП в алюминиевых сплавах, принадлежащих к одному семейству, отличаются незначительно и не зависят от наноструктурирования). Рассчитанные значения производных по давлению КУВП из полученных в [9] экспериментальных значениях КУТП для поликристаллического сплава алюминия $\mathrm{AMg} 6$ и нанокомпозитного сплава $n$ - $\mathrm{AMg} 6 / \mathrm{C}_{60}$, заметно превосходят значения

Таблица 2. Значения производных по давлению КУВП сплавов $\mathrm{AMg} 6$ и $n-\mathrm{AMg} 6 / \mathrm{C}_{60}$, рассчитанные из значений КУТП

\begin{tabular}{c|c|c|c}
\hline Материал & $\partial C_{11} / \partial P$ & $\partial B / \partial P$ & $\partial C_{44} / \partial P$ \\
\hline AMg6 & 27.4 & 20.7 & 5.0 \\
$n$-AMg6/C 60 & 22 & 16.7 & 4.1
\end{tabular}



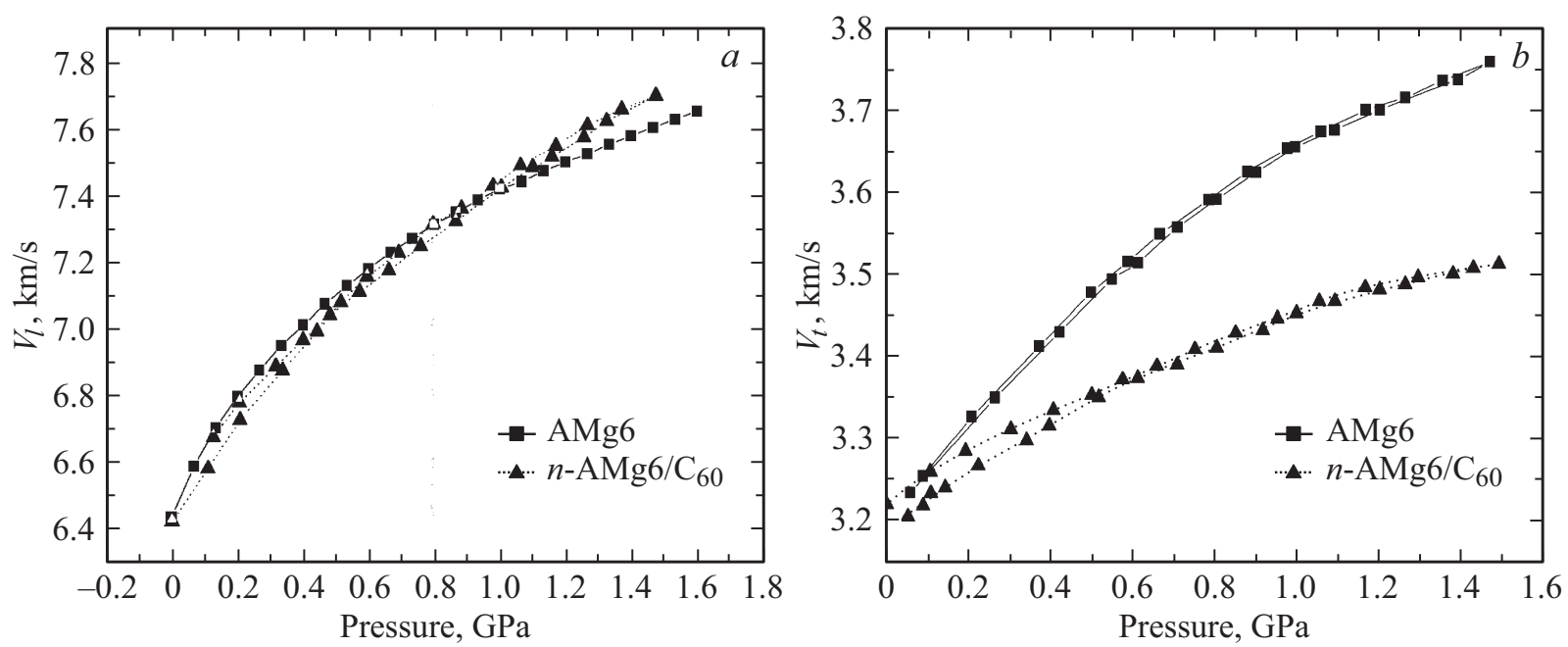

Рис. 1. Зависимости от давлений продольной $\mathbf{V}_{l}(a)$ и поперечной $\mathbf{V}_{t}(b)$ скоростей упругих волн в сплавах $\mathrm{AMg} 6$ и $n-\mathrm{AMg6} / \mathrm{c}_{60}$.

литературных и табличных данных для других алюминиевых сплавов [13]. Поэтому возникла необходимость верификации определенных методом Терстона-Браггера значений КУТП для сплавов $\mathrm{AMg} 6$ и $n$-AMg6/C 60 .

\section{4. Образцы, методики и результаты исследований}

В работе [9] детально описан метод приготовления образцов нанокомпозита $n-\mathrm{AMg} / \mathrm{C}_{60}$. В качестве исходного материала для получения нанокомпозита применялся поликристаллический промышленный сплав алюминия $\mathrm{AMg} 6$, соответствующий ГОСТ 4784-74. Нанокомпозитный алюминиевый сплав получен путем совместного 24-часового размола в шаровой мельнице стружки исходного материала и фуллерита $\mathrm{C}_{60} \mathrm{c}$ последующей консолидацией порошковой смеси методом горячей экструзии.

Из проведенного сравнения значений производных по давлению КУВП для сплавов семейства $\mathrm{Al}-\mathrm{Mg}$, видна зависимость этих значений от содержания $\mathrm{Mg}$ в сплаве. Для более точного определения содержания $\mathrm{Mg}$ в исследуемых сплавах $\mathrm{AMg} 6$ и $n-\mathrm{AMg} 6 / \mathrm{C}_{60}$ мы провели измерения элементного (химического) состава образцов методом дисперсионной рентгеновской спектроскопии, с использованием электронного сканирующего микроскопа JEM 7600F с приставками для энерго-дисперсионного и волнового анализа (EDS и WDS) характеристических спектров химических элементов.

Химический состав исходного сплава AMg6 и нанокомпозитного $n$-АMg6/C 60 представлен в табл. 3 . Снижение процентного содержания $\mathrm{Mg}$ в $n$-AMg6/C 60 произошло, вероятно, за счет увеличения процентного содержания углерода при формировании нанокомпозита $n-\mathrm{AMg} 6 / \mathrm{C}_{60}$.

Измерения зависимостей скоростей ОАВ в образцах сплавов AMg6 и $n$-AMg6/C 60 были проведены ультра- звуковым методом на пьезометрической установке типа „Поршень-цилиндр“ с системой регистрации прошедших и отраженных ультразвуковых сигналов на базе платформы PXI (National Instruments) [14]. Измерения длины пути, проходимого УЗ сигналами измерялись с погрешностью $0.005 \mathrm{~mm}$. Погрешность определения объема образца под давлением составляла несколько процентов. Измерения изменений времени пробега ультразвуковых импульсов продольных ОАВ на частоте $10 \mathrm{MHz}$ и сдвиговых ОАВ на частоте $5 \mathrm{MHz}$ осуществлялось с погрешностью $\pm 0.001 \mu \mathrm{s}$. Погрешность определения давления составила $0.03 \mathrm{GPa}$. Специальные эксперименты были проведены с целью коррекции данных на деформацию камеры с изменением давления и температуры.

Полученные зависимости скоростей продольных и сдвиговых ОАВ и КУВП от давления представлены на рис. 1 и 2 соответственно.

Прежде всего, отметим заметный нелинейный характер зависимостей КУВП от давления. Вероятно, это обусловлено особенностями микроструктуры образцов и генерации давления в камере в начальном диапазоне давлений. Расчеты производных по давлению КУВП проводились для двух диапазонов давления 0.2-0.8 и $0.8-1.6 \mathrm{GPa}$.

На рис. 2 два отрезка со стрелками в верхней части графиков указывают диапазоны давлений, для которых определены производные по давлению упругих модулей образцов сплавов $\mathrm{AMg} 6$ и $n-\mathrm{AMg} 6 / \mathrm{C}_{60}$, а над отрезками приведены их значения.

Таблица 3. Химический состав сплавов $\mathrm{AMg} 6$ и $n-\mathrm{AMg} 6 / \mathrm{C}_{60}$

\begin{tabular}{c|c|c|c|c|c|c}
\hline \multirow{2}{*}{ Alloys } & \multicolumn{5}{|c}{ Chemical composition, at.\% } \\
\cline { 2 - 7 } & $\mathrm{Al}$ & $\mathrm{Mg}$ & $\mathrm{Mn}$ & $\mathrm{O}$ & $\mathrm{C}$ & Total \\
\hline AMg6 & 62.09 & 6.78 & 0.18 & 1.22 & 29.66 & 99.93 \\
n-AMg6/C 60 & 60.63 & 6.31 & 0.26 & 1.35 & 31.38 & 99.93
\end{tabular}



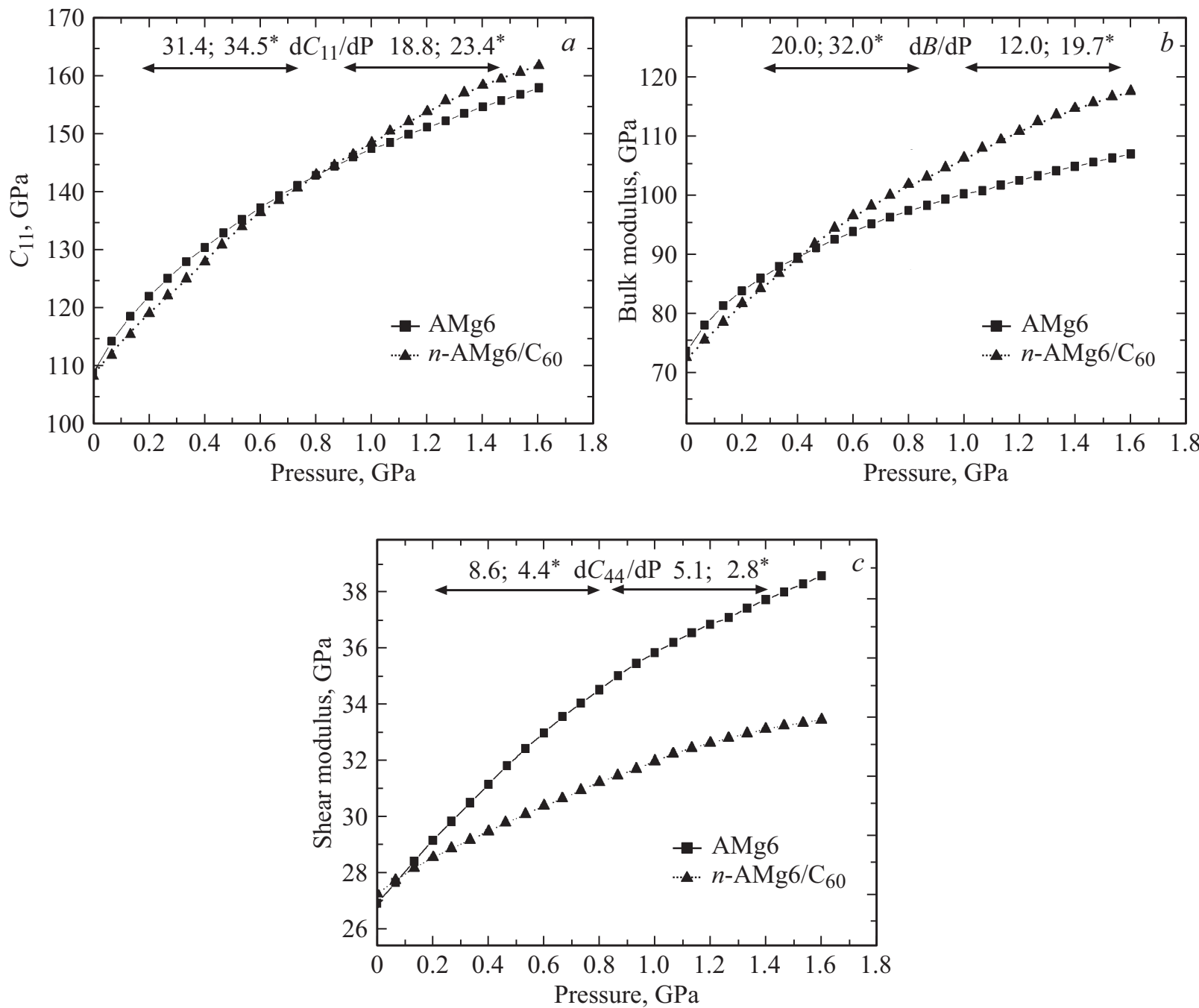

Рис. 2. Зависимости от давления КУВП в сплавах $\mathrm{AMg} 6$ и $n-\mathrm{AMg} 6 / \mathrm{c}_{60}$. Отрезки в верхней части графиков указывают диапазон давлений, в котором определены производные по давлению, их значения для $\mathrm{AMg} 6$ и $n$-AMg6/с60 $\left.{ }^{*}\right)$ указаны над отрезками.

Установленные значения производных по давлению КУВП значительно превосходят известные из литературы данные для алюминиевых сплавов. Согласно этим данным, наибольшими значениями производных обладают алюминиевые сплавы, содержащие магний. В [15] были экспериментально, методом Терстона-Браггера, определены КУТП и рассчитаны производные по давлению модулей упругости алюминиевых сплавов D54S (Mg-4.5\%, Mn-0.8\%, Cr-0.1\%) и B53S (Mg-2.8\%, $\mathrm{Mn}-0.8 \%, \mathrm{Cr}-0.1 \%)$. Сравнение полученных в настоящей работе и известных из литературы данных, приведенных в табл. 4, указывает на наличие зависимости производных по давлению КУВП Мg-содержащих алюминиевых сплавов от концентрации $\mathrm{Mg}$ в сплаве. С привлечением наших данных видно, что эти производные по давлению КУВП монотонно возрастают с увеличением содержания $\mathrm{Mg}$ в сплаве, по крайней мере до концентрации $\mathrm{Mg}$ примерно в 6 at.\%. Для исследованных в настоящей работе сплавов в табл. 4 приведены средние, по двум диапазонам давлений, значения производных по давлению КУВП, а в скобках даны значения, установленные на более линейном участке: в диапазоне давлений 0.8-1.6 GРа. Рассчитанные через КУТП и измеренные в данной работе производные хорошо согласуются между собой.

Таблица 4. Производные по давлению КУВП Мg-содержащих алюминиевых сплавов

\begin{tabular}{|c|c|c|c|c|c|}
\hline Alloys & $\mathrm{Mg}$ (at.\%) & $\partial C_{11} / \partial P$ & $\partial B / \partial P$ & $\partial C_{44} \partial P$ & Источник \\
\hline AMg6 & 6.78 & 27.4 & 20.7 & 5.0 & [9] \\
\hline AMg6 & 6.78 & $25.1(18.8)$ & $16.0(12.0)$ & $6.8(5.1)$ & $\begin{array}{l}\text { В наст. } \\
\text { работе }\end{array}$ \\
\hline n-AMg6 & 6.31 & 22 & 16.7 & 4.1 & [9] \\
\hline$n$-AMg6 & 6.31 & $30.65(23.4)$ & $25.8(19.7)$ & $3.6(2.8)$ & $\begin{array}{l}\text { В наст. } \\
\text { работе }\end{array}$ \\
\hline D54S & 4.5 & 18.4 & 12.8 & 3.5 & {$[14]$} \\
\hline B53S & 2.8 & 9.7 & 6.0 & 2.1 & {$[14]$} \\
\hline
\end{tabular}


Анализ полученных данных указывает, что наноструктурирование приводит к закономерному снижению $\partial C_{44} / \partial P$. Для $\partial C_{11} / \partial P$ и $\partial B / \partial P$ такая закономерность отсутствует и наблюдается значительный разброс значений в обоих диапазонах давлений. Этот результат согласуется с выводами работы [15], согласно которым, для алюминиевых сплавов характерен значительно более широкий разброс значений производных по давлению объемного модуля $\partial B / \partial P$, чем разброс значений $\partial C_{44} / \partial P$, что обусловлено предысторией изготовления и обработки этих сплавов.

Заметим, что в [9] КУТП измерялись при одноосном давлении до $6 \mathrm{MPa}$, а в [15] до $150 \mathrm{MPa}$. Как видно из рис. 2, при малом гидростатическом давлении $(<200 \mathrm{MPa})$ производные по давлению $\partial C_{11} / \partial P$ и дВ $/ \partial P$ для $n$-AMg6 меньше, чем для $\mathrm{AMg6/ \textrm {C } _ { 6 0 }}$.

\section{5. Заключение}

Производные по давлению КУВП в алюминиевом сплаве $\mathrm{AMg} 6$ и нанокомпозите $n-\mathrm{AMg} 6 / \mathrm{C}_{60}$ определены экспериментально с использованием пьезометрической установки высокого давления типа поршень-цилиндр. Измерялись зависимости от давления до $1.6 \mathrm{GPa}$ скоростей продольных и сдвиговых упругих волн, а также плотности образцов, и рассчитывались зависимости от давления КУВП. Установлен нелинейный вид зависимости КУВП от давления. Производные по давлению КУВП вычислялись в диапазонах давлений 0.2-0.8 и $0.8-1.6 \mathrm{GPa}$. Полученные данные сравнивались с рассчитанными для данных материалов по значениям КУТП и данными, известными из литературы для Mg-содержащих алюминиевых сплавов. Полученные результаты хорошо согласуются между собой, что указывает на корректность определения КУТП методом Терстона-Браггера. Установлено, что производные по давлению КУВП Мg-содержащих алюминиевых сплавов монотонно возрастают с увеличением концентрации магния. Наноструктурирование сплава AMg6 приводит с снижению значения производной по давлению модуля сдвига $\left(G=C_{44}\right)$ при незначительных изменениях производных по давлению продольного $\left(C_{11}\right)$ и объемного $(B)$ модулей.

\section{Список литературы}

[1] Л.К. Зарембо, В.А. Красильников. Введение в нелинейную акустику. Наука, М. (1966). 519 с.

[2] О.В. Руденко. УФН 176, 77 (2006).

[3] Конструкционные материалы. Справочник / Под ред. Б.Н. Арзамасова. Машиностроение, М. (1990). 688 с.

[4] Физические величины. Справочник / Под ред. Е.С. Григорьева, Е.3. Мейлихова. Энергоатомиздат, М. (1991). $1232 \mathrm{c}$.

[5] А.И. Коробов, В.М. Прохоров, Д.М. Мехедов. ФТТ 55, 10 (2013).
[6] А.Д. Волков, А.И. Кокшайский, А.И. Коробов, В.М. Прохоров. Акуст. журн. 61, 685 (2015).

[7] N. Lvova, I. Evdokimov, S. Perfilov. Adv. Mater. Res. 1119, 9 (2015).

[8] V. Prokhorov, A. Korobov, A. Kokshaiskii, S. Perfilov, A. Volkov. AIP Conf. Proc. 1685, 080008, (2015).

[9] А.И. Коробов, А.И. Кокшайский, В.М. Прохоров, И.А. Евдокимов, С.А. Перфилов, А.Д. Волков. ФТТ 58, 2384 (2016).

[10] R.N. Thurston, K. Brugger. Phys. Rev. 133. PA1604 (1964).

[11] В.Д. Бланк, В.М. Прохоров, Б.П. Сорокин, Г.М. Квашнин, А.В. Теличко, Г.И. Гордеев. ФТТ 56, 1523 (2014).

[12] D.C. Wallace. In: Solid State Physics / Ed. by H. Ehrenreich, F. Seitz, D. Turnbull. Academic, N. Y. (1970), Vol. 25, 301 p.

[13] И.Н. Францевич, Ф.Ф. Воронов, С.А. Бакута. Упругие постоянные и модули упругости металлов и неметаллов. Наук. думка, Киев (1982). 288 с.

[14] O.V. Stal'gorova, E.L. Gromnitskaya, D.R. Dmitriev, F.F. Voronov. Instrum. Exp. Techn. 39, 880 (1996).

[15] R.T. Smith, R. Stern, R.W.B. Stephens. J. Acoust. Soc. Am. 40. 1002. (1966). 